\title{
Premature Ventricular Contraction Induced Cardiomyopathy Presenting as Trigeminy in the Setting of a Submandibular Abscess and Sepsis
}

\author{
George S Prousi ${ }^{1,2^{*}}$ iD, James A Davis ${ }^{1,2}$, Barry J Feldman ${ }^{2}$ and Sultan Siddique ${ }^{3}$ \\ ${ }^{1}$ University of South Carolina School of Medicine, Columbia, South Carolina, USA \\ ${ }^{2}$ Division of Cardiology, Prisma Health, Columbia, South Carolina, USA \\ ${ }^{3}$ Division of Electrophysiology, Prisma Health, Columbia, South Carolina, USA
}

*Corresponding author: George Stephen Prousi, University of South Carolina School of Medicine; Division of Cardiology, Prisma Health, Columbia, South Carolina, USA

\begin{abstract}
Premature ventricular contractions (PVCs) are often considered benign ectopic heartbeats commonly caused by early depolarizations. Recent attention has been made to the potential manifestation of cardiomyopathy and reduced left ventricular function, especially when PVC burdens exceed 15\% [1]. Further, electrophysiological (EP) studies and ablation have been utilized to identify and potentially eliminate PVC sources with the hope of improving cardiac function and ejection fraction [2]. We report a case of PVC induced cardiomyopathy presenting as trigeminy in the setting of a systemic infection secondary to an oral abscess.
\end{abstract}

\section{Case Presentation}

A 33-year-old female with no significant past medical history presented to the emergency department with 3-days of worsening right sided jaw pain, swelling and associated anorexia. She reported progressive pain and subjective fevers prompting a visit to her primary care physician and subsequently the emergency department. On arrival, vital signs were not able for a temperature of $100.4 \mathrm{~F}$, blood pressure $107 / 62 \mathrm{mmHg}$ and heart rate of 116 BPM. Laboratory evaluation demonstrated WBC 16.9 with a left shift, hemoglobin within normal limits in addition to normal electrolytes including potassium and magnesium. Blood cultures were obtained, and she was initiated on empiric antibiotics with ampicillin/ sulbactam. Oral maxillofacial surgery was consulted and on hospital day one she underwent intraoral incision and drainage of her oral abscess in addition to right molar tooth extraction.

On hospital day two, cardiology was consulted for concern of ongoing tachycardia and observed ventricular ectopy. She was placed on telemetry which revealed trigeminy (Figure 1). An ECG was obtained demonstrating trigeminy with unifocal PVC'swith a left bundle appearance transitioning at the level of $\mathrm{V} 3$ in addition to an inferior direction with positive deflection in II, III and aVF (Figure 2). Further review including repeat laboratory workup did not reveal any abnormalities. On questioning the patient, she reported ongoing symptoms of palpitations that initially began approximately 6-months prior to her presentation. She endorsed intermittent feelings skipped heart beats and stated that for several months, little activity would provoke a rapid heartrate. She denied prior evaluation or workup. She had no family history or cardiomyopathy or sudden cardiac death.

A transthoracic echocardiogram (TTE) revealed an ejection fraction of $25-30 \%$ and a mildly dilated left ventricular cavity without hypertrophy. Wall motion abnormalities were not commented in the setting ongoing PVC burden at the time of image acquisition. Given her new diagnosis of dilated cardiomyopathy, biochemical evaluation including endocrinological, autoimmune, infectious and rheumatological were

Citation: Prousi GS, Davis JA, Feldman BJ, Siddique S (2021) Premature Ventricular Contraction Induced Cardiomyopathy Presenting as Trigeminy in the Setting of a Submandibular Abscess and Sepsis. Int J Clin Cardiol 8:241. doi.org/10.23937/2378-2951/1410241

Accepted: November 13, 2021: Published: November 15, 2021

Copyright: (c) 2021 Prousi GS, et al. This is an open-access article distributed under the terms of the Creative Commons Attribution License, which permits unrestricted use, distribution, and reproduction in any medium, provided the original author and source are credited. 


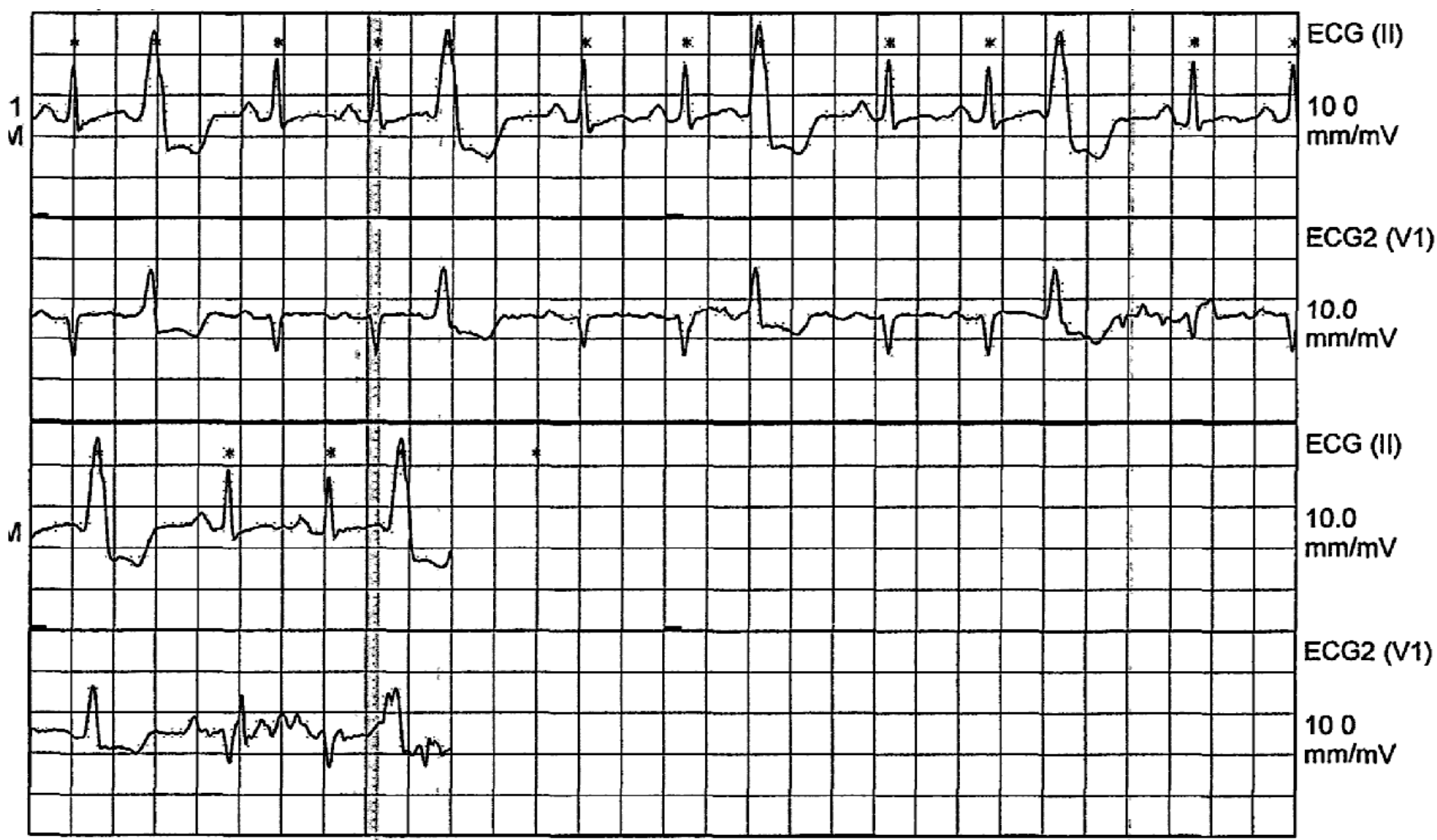

Figure 1: Telemetry tracing showing trigeminy PVC pattern.

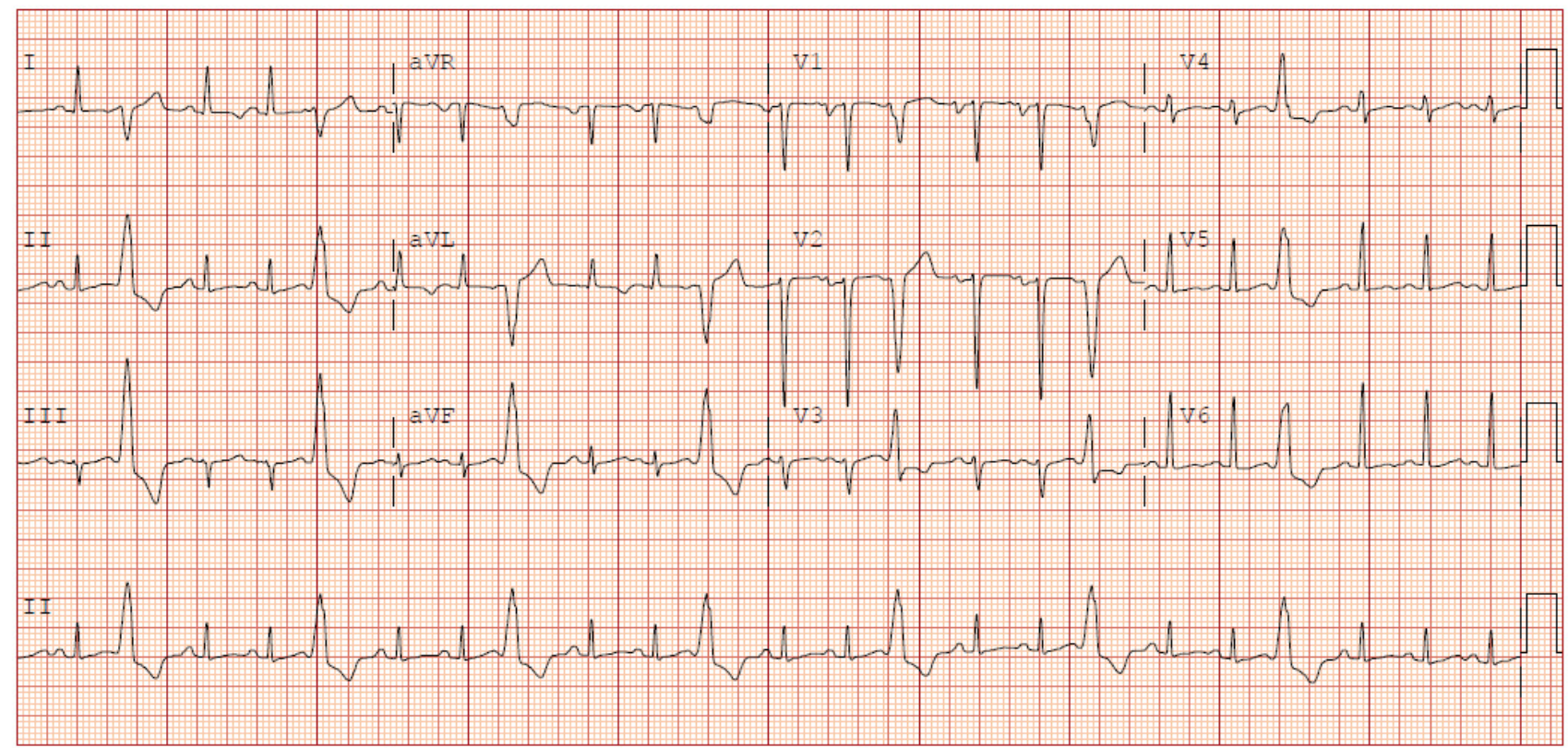

Figure 2: Electrocardiogram suggestive of PVCs originating from the RVOT.

unremarkable. A Holter monitor was placed on discharge with close follow up arranged to determine her PVC burden.

On outpatient evaluation, her cardiac monitor revealed resolution of her trigeminy PVC pattern, however demonstrated a PVC burden of > 30\% and several instances of non-sustained ventricular tachycardia (NSVT). She continued to endorse palpitations in addition to tachycardia, however denied chest pain, orthopnea, paroxysmal nocturnal dyspnea, or dyspnea on exertion. She was initiated on low dose guideline directed medical therapy (GDMT), however was intolerant of beta blockers and an electrophysiology consultation was placed to evaluate likelihood of a PVC induced cardiomyopathy.

Re-evaluation via TTE demonstrated ongoing depressed LV function, however findings were within 3-months of the initiation of her GDMT thereby delaying pursuit of an implantable cardiac defibrillator. Alternatively, an electrophysiology study (EPS) was performed to target and ablate her unifocal PVC origin. 


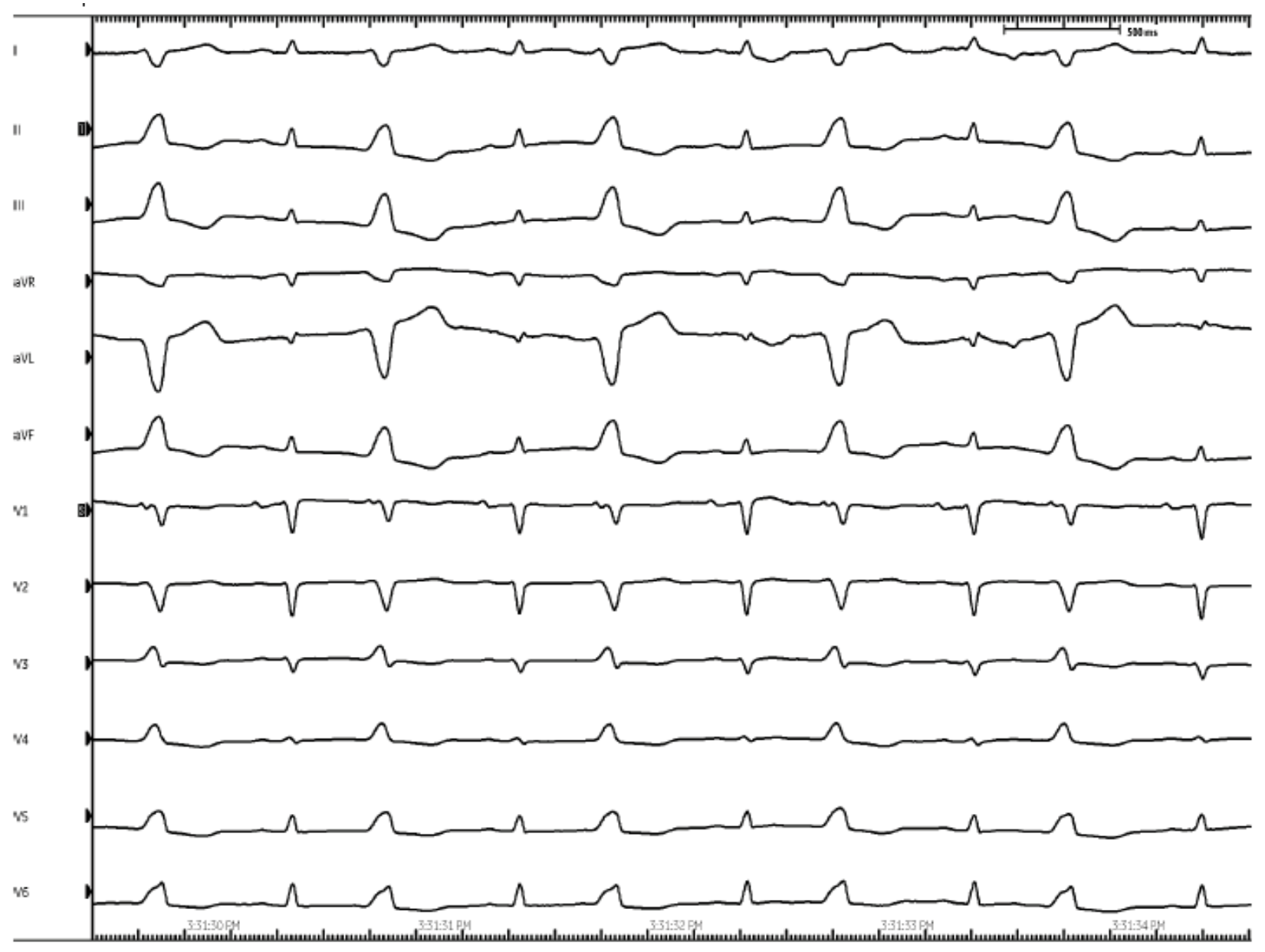

Figure 3: Intracardiac electrocardiogram suggestive of PVCs originating from the RVOT.

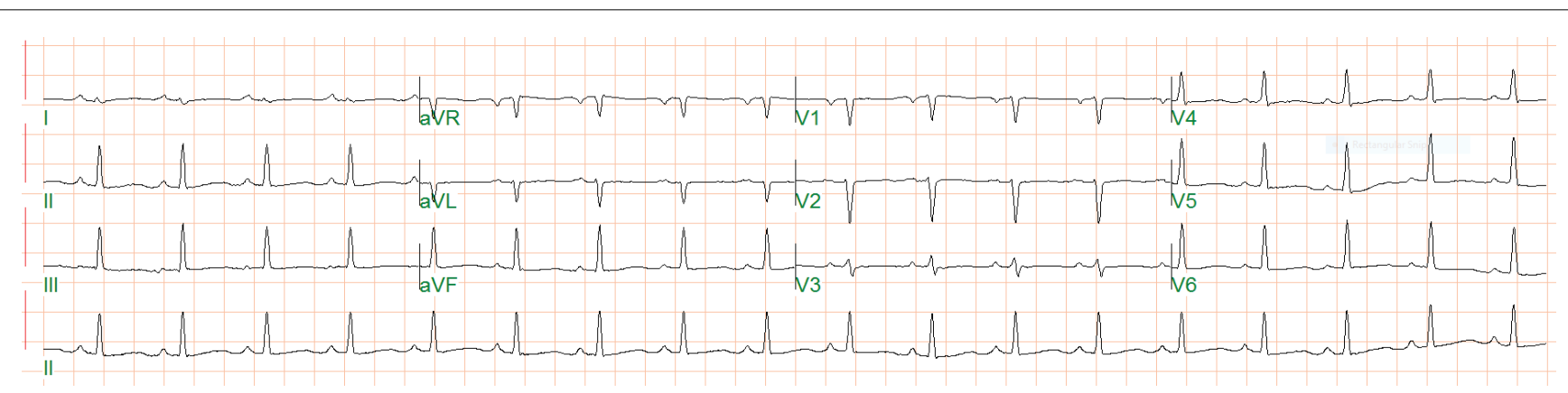

Figure 4: Electrocardiogram demonstrating resolution of PVCs.

Intracardiac echocardiography was utilized for verification of target lesions and real-time ablative probe positioning. A grid mapping catheter localized a bigeminal PVC pattern at the right ventricular outflow tract (RVOT), septally located near the pulmonary valve with a 95\% pacematch (Figure 3). Following localization, radiofrequency ablation was successfully performed at leading to complete resolution of ectopy (Figure 4).

Post-procedure follow-up is currently ongoing and a repeat echocardiogram has yet to demonstrate return to normal systolic function. She is currently not experiencing any PVCs and remains on GDMT. Given her continued reduction in LV function the decision was made to provide Life Vest therapy in hopes of observing cardiac improvement over the next 3 -months. She is currently asymptomatic from a heart failure perspective and endorses baseline functional activity and quality of life.

\section{Discussion}

Premature ventricular complexes (PVCs) are the most common ventricular arrhythmia and are generally considered to be benign in the absence of an associated cardiac condition [3]. PVCs are common and have been observed in up to $75 \%$ of adults increasing in prevalence with age [4]. Cardiomyopathy as it relates to PVC burden has become an increasingly popular area of study despite an unclear mechanism for which it occurs.

Originally thought to be related to a prolonged state of tachycardia, patients with an abnormal number of 
PVCs as compared to the general population interestingly were observed to have similar average heart rates [5]. Given this finding, other speculated mechanisms of PVC induced cardiomyopathy include contractile dysfunction and ventricular desynchrony as described in the context of left bundle branch block $[5,6]$.

Exact quantification of PVC burden lending to LV dysfunction is not well understood though consensus reports have identified a burden of $10-25 \%$ to be associated with the development of cardiomyopathy [7]. Treatment modalities at present include both pharmacological and ablative strategies which are thought to successfully improve systolic function within 4 to 6 months following treatment $[1,8]$.

Currently, AHA/ACC/HRS guidelines consider catheter ablation useful for patients with a declining ventricular function suspected to be due to frequent PVCs (> $15 \%$ ) with one morphological predominance (Class I indication) [9]. Our patient presented with a common form of PVC originating from the RVOT as demonstrated by surface ECG, 24-hour Holter monitoring in addition to intracardiac electrocardiogram allowing for mapping and ablation of one focus.

\section{Conclusion}

PVC induced cardiomyopathy has been evaluated when ectopic burdens exceed $10 \%$ but is commonly evaluated when values are between $15-25 \%$. Historically, PVCs have been thought to be benign, however in the setting of cardiomyopathy, identifying, and ablating the PVC focus has been shown to reverse ventricular dysfunction within several months of ablation.

\section{References}

1. Dukes JW, Dewland TA, Vittinghoff E, Mandyam MC, Heckbert SR, et al. (2015) Ventricular ectopy as a predictor of heart failure and death. J Am Coll Cardiol 66: 101-109.
2. Sharma E, Arunachalam K, Di M, Chu A, Maan A (2017) PVCs, PVC-induced cardiomyopathy, and the role of catheter ablation. Crit Pathw Cardiol 16: 76-80.

3. Gaita F, Giustetto C, Di Donna P, Richardi E, Libero L, et al. (2001) Long-term follow-up of right ventricular monomorphic extrasystoles. J Am Coll Cardiol 38: 364-370.

4. Kennedy HL, Whitlock JA, Sprague MK, Kennedy LJ, Buckingham TA, et al. (1985) Long-term follow-up of asymptomatic healthy subjects with frequent and complex ventricular ectopy. N Engl J Med 312: 193-197.

5. Akoum NW, Daccarett $M$, Wasmund SL, Hamdan $\mathrm{MH}$ (2011) An animal model for ectopy-induced cardiomyopathy. Pacing Clin Electrophysiol 34: 291-295.

6. Gardiwal A, Yu H, Oswald H, Luesebrink U, Ludwig A, et al. (2008) Right ventricular pacing is an independent predictor for ventricular tachycardia/ventricular fibrillation occurrence and heart failure events in patients with an implantable cardioverter-defibrillator. Europace 10: 358-363.

7. Bozkurt B, Colvin M, Cook J, Cooper LT, Deswal A, et al. (2016) Current diagnostic and treatment strategies for specific dilated cardiomyopathies: A scientific statement from the American Heart Association. Circulation 134: e579-e646.

8. Yarlagadda RK, Iwai S, Stein KM, Markowitz SM, Shah BK, et al. (2005) Reversal of cardiomyopathy in patients with repetitive monomorphic ventricular ectopy originating from the right ventricular outflow tract. Circulation 112: 10921097.

9. Al-Khatib SM, Stevenson WG, Ackerman MJ, Bryant WJ, Callans DJ, et al. (2017) 2017 AHA/ACC/HRS guideline for management of patients with ventricular arrhythmias and the prevention of sudden cardiac death: Executive summary: A Report of the American College of Cardiology/ American Heart Association Task Force on Clinical Practice Guidelines and the Heart Rhythm Society. Heart Rhythm 15: e190-e252. 